\title{
Observational Comparison of Star Formation in Different Galaxy Types
}

\author{
Eva K. Grebel ${ }^{1}$ \\ ${ }^{1}$ Astronomisches Rechen-Institut, Zentrum für Astronomie der Universität Heidelberg, \\ Mönchhofstr. 12-14, D-69120 Heidelberg, Germany \\ email: grebel@ari.uni-heidelberg.de
}

\begin{abstract}
Galaxies cover a wide range of masses and star formation histories. In this review, I summarize some of the evolutionary key features of common galaxy types. At the high-mass end, very rapid, efficient early star formation is observed, accompanied by strong enrichment and later quiescence, well-described by downsizing scenarios. In the intermediate-mass regime, early-type galaxies may still show activity in low-mass environments or when being rejuvenated by wet mergers. In late-type galaxies, we find continuous, though variable star formation over a Hubble time. In the dwarf regime, a wide range of properties from bursty activity to quiescence is observed. Generally, stochasticity dominates here, and star formation rates and efficiencies tend to be low. Morphological types and their star formation properties correlate with environment.
\end{abstract}

Keywords. galaxies: formation, galaxies: evolution, galaxies: elliptical and lenticular, galaxies: spiral, galaxies: dwarf, galaxies: stellar content

\section{Introduction}

Star formation differs widely in different galaxy types (e.g., Kennicutt 1998), ranging from slow, low-efficiency events that may be long-lasting to intense, short-duration starbursts. Along the Hubble sequence, typical global present-day star formation rates range from $\sim 0 \mathrm{M}_{\odot} \mathrm{yr}^{-1}$ in giant ellipticals to $\sim 20 \mathrm{M}_{\odot} \mathrm{yr}^{-1}$ in gas-rich, late-type spirals. Starburst galaxies show star formation rates of up to $\sim 100 \mathrm{M}_{\odot} \mathrm{yr}^{-1}$, and ultra-luminous infrared galaxies (ULIRGs) appear to form up to $\sim 1000 \mathrm{M}_{\odot} \mathrm{yr}^{-1}$ in stars. Star formation may be localized or encompass a large fraction of the baryonic gas mass of a galaxy. It may be continuous, declining or increasing in intensity, or episodic. It may be triggered by internal processes within a galaxy or by interactions with other galaxies. Typical sites of present-day star formation in galaxies are located in the extended disks of spirals and irregulars, in the dense gas disks in galaxy centers (circumnuclear star formation), and in regions of compressed gas in starbursts, interacting galaxies, or tidal tails. The star formation histories of galaxies vary with galaxy type, mass, gas content, and environment as well as with time, and are tightly coupled with their chemical evolution.

\section{Very early star formation}

Giant elliptical galaxies are found to have experienced the bulk of their star formation at early times and to have undergone enrichment very rapidly. A similar evolution appears to have taken place in the bulges of spiral galaxies. But how early is "early"? Age dating resolved old stellar field populations in nearby galaxies remains difficult due to crowding, extinction, the superposition of stellar populations of different ages, and the general difficulty of associating individual red giants with a specific age. These problems are exacerbated in galaxies where only integrated-light properties can be analyzed, which makes it very difficult to date stellar populations older than a few Gyr. 


\subsection{Quasars at redshift $\geqslant 6$}

An alternative approach, limited to very luminous and hence presumably very massive, actively star-forming objects, is the analysis of galaxies observed at high redshift. Surprisingly, even the very young quasars discovered at a redshift of $z \sim 6$ (age of the Universe: $\sim 900 \mathrm{Myr}$ ) reveal metal absorption lines that translate into supersolar metallicities, indicating very rapid, early enrichment. These objects may be the precursors of giant ellipticals (Fan et al. 2001). The masses of the central black holes in $z \sim 6$ QSOs are estimated to range from several $10^{8}$ to several $10^{9} \mathrm{M}_{\odot}$. This suggests formation redshifts of more than 10 for putative $100 \mathrm{M}_{\odot}$ seed black holes if continuous Eddington accretion is assumed. Other, more rapid black hole formation mechanisms may also play a role (e.g., Fan 2007).

For one of the highest-redshift quasars known, SDSS J114816.16+525150.3 at $z=6.42$, sub-millimeter and radio observations suggest a molecular gas mass $(\mathrm{CO})$ of $\sim 5 \times 10^{10}$ $\mathrm{M}_{\odot}$ within a radius of $2.5 \mathrm{kpc}$ around the central black hole if the gas is bound. The star formation rate in this object is $\sim 1000 \mathrm{M}_{\odot} \mathrm{yr}^{-1}$, akin to what has been derived for star-bursting ultraluminous infrared galaxies. Even within $20 \mathrm{kpc}$, the inferred mass estimate for the QSO's host is still comparatively low, $\sim 10^{11} \mathrm{M}_{\odot}$. A central massive $10^{12}$ $\mathrm{M}_{\odot}$ bulge has evidently not yet formed (Walter et al. 2004; Fan 2007).

In several $z \gtrsim 6$ QSOs dust has been detected. One of the primary sources for dust in the present-day Universe are low- and intermediate-mass asymptotic giant branch stars, but these need at least some 500 Myr to 1 Gyr to begin generating dust in their envelopes. In quasars in the early Universe, other mechanisms are believed to be responsible, including dust production in supernovae of type II (Maiolino et al. 2004) and in quasar winds (Elvis et al. 2002). Recently two $z \sim 6$ QSOs without dust were detected, which indicates that these objects are probably first-generation QSOs forming in an essentially dust-free environment (Jiang et al. 2010). This discovery illustrates that even at these very early times of less than 1 Gyr after the Big Bang, galactic environments differ in the onset of massive star formation and in the degree of heavy-element pollution, with some regions already having experienced substantial enrichment. One may speculate that the densest regions are the first ones to start massive star formation, and that the dusty high-redshift QSOs trace these regions.

Magnesium, an $\alpha$ element, is produced in supernovae of type II and hence expected to be generated soon after massive star formation commences. Iron is predominantly produced in supernovae of type Ia, requiring a minimum delay time of $~ 300$ Myr when instantaneous starbursts are considered (e.g., Matteucci \& Recchi 2001). Fe is detected in $z \sim 6$ QSOs despite their young age. The FeII/Mg II ratio (which is essentially a proxy for $\mathrm{Fe} / \alpha$ ) is found to be comparable to that of lower-redshift QSOs (e.g., Barth et al. 2003; Freudling et al. 2003; Kurk et al. 2007) and to be around solar or super-solar metallicity. The near-constancy of the Fe II/Mg II ratio as a function of redshift implies a lack of chemical evolution in QSOs since $z \sim 6$ and suggests a formation redshift of the SN Ia progenitors of $z \gtrsim 10$. Extremely rapid enrichment on a time scale of just a few hundred Myr must have occurred in these QSOs, much faster than the slow enrichment time scales of spiral galaxies. Nonetheless, also here evolutionary differences are becoming apparent: Some of the $z \sim 6$ QSOs are less evolved, not showing strong Fe II emission lines (Iwamuro et al. 2004) and hence no significant SN Ia contributions yet.

\subsection{Galaxies at redshift $\geqslant 7$}

Moving to even higher redshifts, the analysis of spectral energy distributions obtained from near-infrared imaging data of galaxies at redshifts of 7 (age of the Universe $\sim 770 \mathrm{Myr}$ ) and 8 ( $\sim 640 \mathrm{Myr})$ revealed median ages of $\sim 200 \mathrm{Myr}$ for their stellar populations, but 
even younger ages are not excluded (Finkelstein et al. 2010). The typical stellar masses of the galaxies at $z \sim 7$ are $<10^{9} \mathrm{M}_{\odot}$; they may be as low as only $10^{7} \mathrm{M}_{\odot}$ at $z \sim 8$. While some galaxies are consistent with having no internal dust extinction, the median value is $A_{V} \sim 0.3 \mathrm{mag}$. Finkelstein et al. (2010) estimate that some $10^{6} \mathrm{M}_{\odot}$ of dust may have been produced in these galaxies through massive $\left(12-35 \mathrm{M}_{\odot}\right)$ stars that turned into SNe II, a process expected to take less than 20 Myr (Todini \& Ferrara 2001).

Finkelstein et al. (2010) derive metallicities of $0.005 Z_{\odot}$ for the majority of their galaxies, while some may have somewhat higher values $\left(0.02 Z_{\odot}\right)$. Inferring maximal stellar masses of a few times $10^{9} \mathrm{M}_{\odot}$ for the $z \sim 7$ and 8 galaxies, the authors emphasize that these masses are still considerably lower than suggested for $L_{\star}$ counterparts at $z<6$. These distant, luminous galaxies whose colors resemble those of local, metal-poor star-bursting dwarf galaxies trace the earliest times of high-redshift galaxy formation accessible to us with the current instrumentation.

\section{Global evolutionary trends}

Global evolutionary trends in galaxy evolution over cosmic times (e.g., Madau et al. 1996) are best inferred from individual or combined galaxy surveys covering a wide redshift range. Marchesini et al. (2009) derive the redshift evolution of the global stellar mass density for galaxies with stellar masses in the range of $10^{8}<\mathrm{M}_{\star} / \mathrm{M}_{\odot}<10^{13}$. Galaxies with lower stellar masses do not appear to contribute significantly to the mass density budget. Marchesini et al. find that approximately $45 \%$ of the present-day stellar mass was generated from $3>z>1$ (within 3.6 Gyr). From $z \sim 1$ to the present, i.e., during the last $\sim 7.5 \mathrm{Gyr}$, the remaining $50 \%$ were produced.

\subsection{Downsizing}

Considering cosmic star formation histories, there is compelling evidence for "downsizing" (Cowie et al. 1996): The stars in more massive galaxies usually formed at earlier cosmic epochs and over a shorter time period. For a pedagogical illustration, see Fig. 9 in Thomas et al. (2010). High-redshift galaxies tend to have star formation rates higher than those found in the local Universe. Also, these galaxies are typically more massive than lowredshift star-forming galaxies (Seymour et al. 2008).

Juneau et al. (2005) show that the star formation rate density depends strongly on the stellar mass of galaxies. For massive galaxies with $\mathrm{M}_{\star}>10^{10.8} \mathrm{M}_{\odot}$ the star formation rate density was about six times higher at $z=2$ than at the present, remaining approximately constant since $z=1$. The star formation rate density in their "intermediate-mass" bin, $10^{10.2} \leqslant \mathrm{M}_{\star} / \mathrm{M}_{\odot} \leqslant 10^{10.8} \mathrm{M}_{\odot}$, peaks at $z \sim 1.5$, whereas since $z<1$ most of the activity was in lower-mass galaxies (Juneau et al. 2005).

For quasar host galaxies observed with the Herschel satellite, Serjeant et al. (2010) find that high-luminosity quasars have their peak contribution to the star formation density at $z \sim 3$, while the maximum contribution of low-luminosity quasars peaks between $1<z<2$. The authors suggest that this indicates a decrease in both the rate of major mergers and in the gas available for star formation and black hole accretion.

\subsection{Mass-metallicity relations}

From an analysis of $\sim 160,000$ galaxies in the local Universe observed by the Sloan Digital Sky Survey (SDSS), Gallazzi et al. (2008) conclude that approximately $40 \%$ of the total amount of metals contained in stars is located in bulge-dominated galaxies with predominantly old populations. Disk-dominated galaxies contain $<25 \%$, while both types of galaxies contribute similarly to the total stellar mass density. 
Panter et al. (2008) derived the mass-fraction-weighted galaxian metallicity as a function of present-day stellar mass based on the analysis of $>300,000$ galaxies from the SDSS. Panter et al. find a flat relation with little scatter $(\sim 0.15$ dex $)$ around $\sim 1.1 Z_{\odot}$ for galaxies with masses $\gtrsim 10^{10.5} \mathrm{M}_{\odot}$. Below $\sim 10^{10} \mathrm{M}_{\odot}$ there is a clear trend of decreasing metallicity of about 0.5 dex per dex decline in mass, although the dispersion is relatively large $(\sim 0.5 \mathrm{dex})$. When considering only (the very few) galaxies in which more than $50 \%$ of the light comes from populations younger than $500 \mathrm{Myr}$, only systems with stellar masses $<10^{10} \mathrm{M}_{\odot}$ contribute significantly - another indication of downsizing.

\subsection{Environmental trends}

Galaxies in high-density regions are generally found to have higher metallicities than those in low-density regions (see, e.g., Panter et al. 2008). Sheth et al. (2006) find that galaxies with above-average star formation rates and high metallicities at high redshifts are situated mainly in galaxy clusters in the present-day Universe. Interestingly, Poggianti et al. (2010) infer that high-redshift clusters were denser environments with respect to both galaxy number and mass than contemporary clusters, which might have fostered the intense star-formation activity and growth in their most massive galaxies. The clustering strength of star-forming galaxies decreases with decreasing redshift (Hartley et al. 2010). Galaxies that are passively evolving at the present time (typically galaxies with halo masses $>10^{13} \mathrm{M}_{\odot}$ ) are twice as strongly clustered than present-day star-forming galaxies (which are typically at least a factor of 10 less massive).

Sheth et al. (2006) point out that at lower redshifts, star formation (either in terms of mass or fraction) is anti-correlated with environment such that dense environments have shown lower star formation rates than low-density regions during the past $\sim 5$ Gyr. Interestingly, in the present-day Universe star-forming galaxies appear to evolve fairly independently of their environment, with intrinsic properties playing a determining role (Balogh et al. 2004; Poggianti et al. 2008). Overall, environment seems to have had little influence on the cosmic star formation history since $z<1$ (Cooper et al. 2008).

The famous morphology-density relation in clusters and groups (Oemler 1974; Dressler 1980; Postman \& Geller 1984) describes the increase in the fraction of ellipticals with galaxy density, while the fraction of spirals declines. Similarly, there is a pronounced correlation of morphological types with cluster-centric radius (Whitmore et al. 1993) such that in the innermost regions of a cluster the fraction of ellipticals shows a strong increase, while the fraction of spirals drops sharply. The S0 fraction rises less steeply with decreasing radius and also drops in the innermost regions. These relations are interpreted as suggestive of the growth of ellipticals (and S0s) in high-density regions at the expense of spirals. Goto et al. (2003) find three different regimes depending on galaxy density: For densities $<1 \mathrm{Mpc}^{-2}$, the morphology-density and morphology-radius relations become rather weak. For $1-6 \mathrm{Mpc}^{-2}$, the fraction of late-type disks decreases with clustercentric radius, while early-type spiral and S0 fractions increase. For the highest-density clusters with $>6 \mathrm{Mpc}^{-2}$, also these intermediate-type fractions decrease with clustercentric radius, while the early-type fractions increase.

For dwarf galaxies, similar global morphology-density and morphology-radius trends are observed in the sense that gas-deficient early-type dwarfs tend to concentrate within $\sim 300 \mathrm{kpc}$ around massive galaxies in groups or are predominantly found in the inner regions of clusters, while gas-rich late-type dwarfs are found in the outskirts and in the field (e.g., Grebel 2000; Karachentsev et al. 2002a, 2003a; Lisker et al. 2007). The poorest low-density groups are dominated by late-type galaxies both among their massive and their dwarf members (Karachentsev et al. 2003a, 2003b), while in richer, more compact groups the early-type fractions increase also among the dwarfs, as does the morphological 
segregation (see, e.g., Karachentsev et al. 2002a, 2002b). Various physical mechanisms including ram pressure and tidal stripping are discussed to explain apparent evolutionary connections of dwarf galaxies with environment (e.g., van den Bergh 1994; Vollmer et al. 2001; Grebel et al. 2003; Dong et al. 2003; Hensler et al. 2004; Kravtsov et al. 2004; Mieske et al. 2004; Lisker et al. 2006; Mayer et al. 2006; D’Onghia et al. 2009).

\section{Massive early-type galaxies}

Analyzing a volume-limited sample of $>14,000$ early-type galaxies from the SDSS, Clemens et al. (2009a) find that their ages, metallicities, and $\alpha$-element enhancement increase with their mass (using velocity dispersion, $\sigma$, as indicator). For galaxies with $\sigma>180 \mathrm{~km} \mathrm{~s}^{-1}$, the mean age decreases with decreasing galactocentric radius, while the metallicity increases. Clemens et al. suggest that the massive early-type galaxies were assembled at $z \lesssim 3.5$, merging with low-mass halos that began to form at $z \sim 10$. These subhalos contributed older, metal-poor stars that are still distributed over large radii. Gas-rich mergers, very frequent at early times, contributed fuel for intense star formation in the central regions of the galaxies, while mergers at later times were increasingly gaspoor or dry. Clemens et al. find these radial age and metallicity gradients in early-type galaxies regardless of environment, although massive ellipticals in clusters are on average $\sim 2$ Gyr older than those in the field, supporting the trends expected for downsizing.

\subsection{Environment and rejuvenation}

From an analysis of Spitzer Space Telescope (SST) data of 50 early-type galaxies in the Coma cluster, Clemens et al. (2009b) find that while the majority is passive, some $\sim 30 \%$ of the galaxies are either younger than 10 Gyr or were rejuvenated in the last few Gyr.

Combining near-UV photometry from the Galaxy Evolution Explorer (GALEX) satellite with SDSS data of a volume-limited sample of 839 luminous early-type galaxies, Schawinski et al. (2007) conclude that $\sim 30 \%$ of these objects show evidence of recent $(<1$ Gyr) star formation ( $29 \%$ ellipticals, $\sim 39 \%$ lenticulars $)$. Moreover, they show that that low-density environments contain $\sim 25 \%$ more UV-bright early-type galaxies.

Thomas et al. (2010) analyze low-redshift > 3000 early-type galaxies from the SDSS and infer that intermediate-mass and low-mass galaxies show evidence for a secondary peak of more recent star formation around $\sim 2.5$ Gyr ago. They find that the fraction of these rejuvenated galaxies becomes larger with decreasing galaxy mass and with decreasing environmental density, reaching up to $45 \%$ at low masses and low densities. Thomas et al. conclude that the impact of environment increases with decreasing galaxy mass via mergers and interactions and has done so since $z \sim 0.2$.

\section{2. $E+A$ galaxies}

An interesting class of rejuvenated early-type galaxies are the so-called "E+A" galaxies, ellipticals that show the typical K-star spectra with $\mathrm{Mg}$, Ca, and Fe absorption lines as well as strong Balmer lines akin to A-stars (Dressler \& Gunn 1983), indicating that in addition to the usual passive evolution, they experienced star formation within the last Gyr. The absence of [O II] and $\mathrm{H} \alpha$ emission lines shows that there is no ongoing star formation. These post-starburst galaxies are observed both in clusters and in the field.

SDSS studies support suggestions that the $\mathrm{E}+\mathrm{A}$ phenomenon is created by interactions and/or mergers. About $30 \%$ of the E+A galaxies show disturbed morphologies or tidal tails (Goto 2005). The analysis of $660 \mathrm{E}+\mathrm{A}$ galaxies revealed that these objects have a $54 \%$ higher probability of having close companion galaxies than normal galaxies $(\sim 8 \%$ vs. $\sim 5 \%$; Yamauchi et al. 2008). 


\section{Spirals and irregulars}

\subsection{Stellar halos of disk galaxies}

Spiral galaxies usually have extended, low-density stellar halos (Zibetti et al. 2004) whose density decreases with $\sim r^{-3}$. In the Milky Way the stellar halo consists of old, metalpoor stars and globular clusters on eccentric prograde or retrograde orbits (see Freeman \& Bland-Hawthorn 2002 and Helmi 2008 for recent reviews of the Galactic halo). As many of half of the field stars in the halo may have originated in disrupted globular clusters (Martell \& Grebel 2010; see Odenkirchen et al. 2001a for an example).

$\Lambda \mathrm{CDM}$ simulations suggest that in galaxies with few recent mergers the fraction of halo stars formed in situ amounts to $20 \%$ to $50 \%$ (Zolotov et al. 2009). Johnston et al. (2008) propose that halos dominated by very early accretion show higher $[\alpha / \mathrm{Fe}]$ ratios, whereas those that accreted mainly high-luminosity satellites should exhibit higher $[\mathrm{Fe} / \mathrm{H}]$.

The detection of substructure (e.g., Newberg et al. 2002; Yanny et al. 2003; Bell et al. 2008) as well as chemical and kinematic signatures (Carollo et al. 2007; Geisler et al. 2007) support the scenario that part of the Galactic halo was accreted. The individual stellar element abundance patterns suggest that such accretion may have mainly occurred at very early times, since the $[\alpha / \mathrm{Fe}]$ ratios of metal-poor halo stars match the ones found in similar stars in the Galactic dwarf spheroidal and irregular companions (e.g., Koch et al. 2008a). Prominent morphological evidence of ongoing dwarf galaxy accretion has been found not only in the Milky Way (e.g., Ibata et al. 1994), but also around other spiral galaxies (e.g., Ibata et al. 2001; Zucker et al. 2004; Martínez-Delgado et al. 2008).

\subsection{Bulges of disk galaxies and formation scenarios}

Early-type spirals show prominent bulges, which become less pronounced and ultimately vanish in late-type spirals and irregulars. Classical bulges (found in early-type to Sbc spirals) resemble elliptical galaxies in their properties, are dominated by old, mainly metal-rich stars with a large metallicity spread, show hot stellar kinematics, and follow a de Vaucouleurs surface brightness profile just like typical elliptical galaxies. Pseudobulges (in disk galaxies later than Sbc) resemble disk galaxies, have similar exponential profiles, are rotation-dominated, and may contain a nuclear bar, ring, or spiral. They are believed to form from disk material via secular evolution (Kormendy \& Kennicutt 2004). A recent analysis combining data from SST and GALEX found that all bulges show some amount of ongoing star formation, regardless of their type (Fisher et al. 2009), with small bulges having formed 10 to $30 \%$ of their mass in the past 1 to 2 Gyr (Thomas \& Davies 2006). Extracting a sample of $>3000$ nearby edge-on disk galaxies from the SDSS, Kautsch et al. (2006) showed that approximately $30 \%$ of the edge-one galaxies are bulge-less disks.

Noguchi (1999) suggested that massive clumps forming at early times in galactic disks move towards the galactic center due to dynamical friction, merge, and form the galactic bulge. This scenario leads to the observed trend of increased bulge-to-disk ratios with increased total galactic masses. van den Bergh (2002) noted that while most of the galaxies observed in the Hubble Deep Fields at $z<1$ have disk-like morphologies, most galaxies at $z>2$ look clumpy or chaotic. Analyzing such "clump clusters" and "chain galaxies" in the Hubble Ultra Deep Field, Elmegreen et al. (2009) find that the masses of the star-forming clumps are of the order to $10^{7}$ to $10^{8} \mathrm{M}_{\odot}$. Bournaud et al. (2007) argue that clusters of such massive, kpc-sized clumps can form bulges in less than 1 Gyr, while the system as a whole evolves from a violently unstable disk into a regular spiral with an exponential or double exponential disk profile on a similarly rapid time scale. While the coalescence of these clumps resembles a major merger with respect to orbital 
mixing, the resulting bulge has no specific dark-matter component, which distinguishes it from bulges formed via galaxy mergers (Elmegreen et al. 2008).

\subsection{Disks and long-term evolutionary trends for disk galaxies}

Disks are the primary sites of present-day star formation in spiral galaxies, and it seems likely that they have continued to form stars for a Hubble time. Disks show ordered rotation, and their stars move around the galactic center on near-circular orbits. The rotational velocities greatly exceed the velocity dispersion by factors of 20 or more.

Gas-deficient early-type disk galaxies show little activity at the present time, while gasrich late-type disks experience wide-spread, active star formation. Star formation occurs mainly in the midplane of the thin disks, in particular along spiral arms, where recent events are impressively traced by giant H II regions. Spiral density waves may induce star formation (see Martínez-García et al. 2009; and references therein), although it has been suggested that this mechanism may contribute less than $50 \%$ to the overall star formation rate (Elmegreen \& Elmegreen 1986).

In the Milky Way, the star formation in the disk was not constant, but shows extended episodes of increased and reduced activity (e.g., Rocha-Pinto et al. 2000), a radial metallicity gradient, a G-dwarf problem, and a large metallicity scatter at all ages (Nordström et al. 2004). The thin disk is embedded in a lower-density, kinematically hotter stellar population consisting of older, more metal-poor stars - the thick disk (Gilmore \& Reid 1983; Bensby et al. 2005). The chemical similarity of Galactic bulge and thick disk stars might suggest that the Milky Way does not have a classical bulge (Meléndez et al. 2008).

Dalcanton \& Bernstein (2002) showed that thick disks are ubiquitous also in bulge-less late-type disk galaxies, which indicates that their formation is a universal property of disk formation independent from the formation of a bulge. A variety of mechanisms for the formation of thick disks has been proposed, including formation from accreted satellites, gas-rich mergers, heating of an early thin disk by mergers, heating via star formation processes, and radial migration (e.g., Wyse et al. 2006; Brook et al. 2004; Kroupa 2002; Bournaud et al. 2009; Roškar et al. 2008). Sales et al. (2009) suggest that the eccentricity distribution of thick disk stars may permit one to distinguish between these scenarios.

In cosmological simulations disk galaxies may form, for example, via major, wet mergers (see, e.g., Barnes 2002; Governato et al. 2009) or without mergers via inside-out and vertical collapse in a growing dark matter halo (Samland \& Gerhard 2003).

As noted by van den Bergh (2002) based on an analysis of the Hubble Deep Fields, roughly one third of the objects at $z>2$ seem to be experiencing mergers. He suggests that from $1<z<2$ a transition from merger-dominated to disk-dominated star formation occurred. Moreover, he finds that at $z>0.5$, there are fewer and fewer barred spirals. While early-type galaxies assume their customary morphologies relatively early on, $46 \%$ of the spirals at $0.6<z<0.8$ are still peculiar, and with higher redshift, the spiral arm patterns become increasingly chaotic. Also within the class of spiral galaxies there are trends: Only $\sim 5 \%$ of the Sa and Sab galaxies are peculiar at $z \sim 0.7$, while almost $70 \%$ of the Sbc and Sc types are still peculiar.

Elmegreen et al. (2007) suggest that the formation epoch of clumpy disk galaxies may extend up to $z \sim 5$. The ones experiencing major mergers may form red spheroidals at $2 \lesssim z \lesssim 3$, whereas the others evolve into spirals. Elmegreen et al. propose that the the star formation activity in clumpy disks is caused by gravitational collapse of portions of the disk gas without requiring an external trigger.

Regarding environment, Poggianti et al. (2009) find that the fraction of ellipticals remains essentially constant below $z=1$, while the spiral and S0 fractions continue to evolve, showing the most pronounced evolution in low-mass galaxy clusters. They 
attribute this to secular evolution and to environmental mechanisms that are more effective in low-mass environments. At low redshifts, the declining spiral fraction with density is driven by late-type spirals (Sc and later; Poggianti et al. 2008).

\subsection{Irregulars}

Irregular galaxies are gas-rich, low-mass, metal-poor galaxies without spiral density waves, which show recent or ongoing star formation that appears to have extended over a Hubble time (Hunter 1997). Many studies found the H I gas to be considerably more extended than the stellar component in irregulars (e.g., Young \& Lo), but more recent, deep optical surveys show that the optical extent of at least some of these galaxies has been underestimated (e.g., Kniazev et al. 2009). All nearby irregulars and dwarf irregulars have been found to contain old populations, although their fractions differ (Grebel \& Gallagher 2004). The old populations tend to be more extended than the more recent star formation (e.g., Minniti \& Zijlstra 1996; Kniazev et al. 2009) and show a more regular distribution (e.g., Zaritsky et al. 2000; van der Marel 2001).

Irregulars are usually found in the outskirts of groups and clusters or in the field, thus interactions with other galaxies are likely to be rare. Their star formation appears to be largely governed by internal processes and seems to be stochastic. Rather than experiencing brief, intense starbursts, irregulars typically show extended episodes of star formation interrupted by short quiescent periods - so-called gasping star formation (e.g., Cignoni \& Tosi 2010). The long-term star formation amplitude variations amount to factors of 2 to 3 (Tosi et al. 1991). For a review of irregulars and dwarf irregulars in the Local Group, for which we have the most detailed data to date, see Grebel (2004).

While the more massive irregulars are rotationally supported and show solid-body rotation, low-mass dwarf irregulars are dominated by random motions. Star formation ceases at lower gas density thresholds than in spirals (e.g., Parodi \& Binggeli 2003), and the global gas density of the highly porous interstellar medium has been found to lie below the Toomre criterion for star formation (van Zee et al. 1997). Turbulence may create local densities exceeding the star formation threshold (e.g., Stanimirovic et al. 1999). Low-mass dwarf irregulars without measurable rotation show less centrally concentrated star formation and have lower star formation rates (Roye \& Hunter 2000; Parodi \& Binggeli 2003). In most irregulars, star formation occurs within the galaxies' Holmberg radius and within three disk scale lengths (Hunter \& Elmegreen 2004).

In contrast, in blue compact dwarf (BCD) galaxies the highest star formation rates are found, and star formation occurs mainly in the central regions (Hunter \& Elmegreen). (We do not discuss BCDs and other gas-rich dwarfs in more detail here. For an overview of different dwarf types and their properties, see Grebel (2003)).

Once believed to be chemically homogeneous, there is now evidence of metallicity variations at a given age in several irregulars (e.g., Kniazev et al. 2005; Glatt et al. 2008). This suggests that local processes dominate the enrichment and that mixing is not very efficient. Irregulars follow a fairly well-defined metallicity-luminosity relation, which however is offset from that of early-type dwarfs covering the same luminosity range. Surprisingly, the offset is such that the continuously star-forming irregulars and dwarf irregulars have lower metallicities at a given luminosity than the inactive early-type dwarfs (e.g., Richer et al. 1998), a discrepancy that holds even when comparing stellar populations of the same age (Grebel et al. 2003). Taken at face value, this may imply that the enrichment of irregulars was less efficient and slower than that of early-type dwarfs. BCDs and in particular extremely metal-deficient galaxies continue this trend and appear to be too luminous for their present-day, low metallicities even when compared to normal irregulars (Kunth \& Östlin 2000; Kniazev et al. 2003). 


\subsection{Star formation "demographics"}

Lee et al. (2007) investigate the star formation "demographics" of star-forming galaxies out to $11 \mathrm{Mpc}$ combining $\mathrm{H} \alpha$ and GALEX UV fluxes. Their sample includes spirals, irregulars, and BCDs. Lee et al. identify three different star formation regimes:

1. Galaxies with maximum rotational velocities $V_{\max }>120 \mathrm{~km} \mathrm{~s}^{-1}$, total B-band magnitudes of $M_{B} \lesssim-19$, and stellar masses $\gtrsim 10^{10} \mathrm{M}_{\odot}$ are mainly bulge-dominated galaxies with relatively low specific star formation rates and increased scatter in these rates. Also the mass-metallicity relation changes its slope in this regime (Panter et al. 2007), and supernova ejecta can be retained (Dekel \& Woo 2003). Bothwell et al. (2009) find that the $\mathrm{H}$ I content of these massive galaxies decreases faster than their star formation rates, leading to shorter $\mathrm{H}$ I consumption time scales and making the lack of gas a plausible reason for the observed quenching of star formation activity.

2. Galaxies with $\sim 120 \mathrm{~km} \mathrm{~s}^{-1}>V_{\max }>50 \mathrm{~km} \mathrm{~s}^{-1}$ and $-19<M_{B}<-15$ comprise mainly late-type spirals and massive irregulars. Lee at al. (2007) suggest that spiral structure acts as an important regulatory factor for star formation. They find that the galaxies in this intermediate-mass regime exhibit a comparatively tight, constant relation between star formation rate and luminosity (or rotational velocity). The star formation rates show fluctuations of 2 to 3 , and the current star formation activity is about half of its average value in the past. Bothwell et al. (2009) argue that the galaxies in this regime evolve secularly. They show that the star formation rates decrease with the galaxies' H I mass, and that the H I consumption time scales increase with decreasing luminosity.

3. Below $V_{\text {max }}=50 \mathrm{~km} \mathrm{~s}^{-1}$ and $M_{B}>-15$, dwarf galaxies, particularly irregulars, dominate. At these low masses, the star formation rates exhibit much more variability ranging from significantly higher (e.g., in BCDs) to significantly lower (e.g., in so-called transition-type dwarfs with properties in between dwarf irregulars and dwarf spheroidal galaxies, see Grebel et al. 2003) star formation activity than in the higher-mass regimes. Overall, there is a general trend towards lower star formation rates. Stochastic intrinsic processes, feedback, and the ability to retain gas play an important role here. Bothwell et al. (2009) find that for many of the galaxies in the low-mass regime the $\mathrm{H}$ I consumption time scale exceeds a Hubble time (in good agreement with the results of Hunter 1997).

Bothwell et al. show that the $\mathrm{H}$ I consumption time scales have a minimum duration of more than $100 \mathrm{Myr}$. They argue that this minimum duration corresponds to the gas mass divided by the minimum gas assembly time, i.e., the free-fall collapse time.

\section{Early-type dwarfs}

We end this review by summarizing the star formation properties of early-type dwarfs, most notably of dwarf ellipticals (dEs) and dwarf spheroidals (dSphs; see Grebel 2003; Grebel et al. 2003). Typically located in high-density regions such as the immediate surroundings of massive galaxies or in galaxy clusters, this dense environment may have affected the evolution of these now gas-deficient galaxies (Section 3.3). Structural and kinematic studies suggest that early-type dwarfs are strongly dark-matter dominated (e.g., Odenkirchen et al. 2001b; Klessen et al. 2003; Wilkinson et al. 2004; Koch et al. 2007a, 2007b; Walker et al. 2007; Gilmore et al. 2007; Wolf et al. 2010), and there are even indications of a constant dark-matter halo surface density from spirals to dwarfs (Donato et al. 2009). However, it is not yet clear whether the apparently constant total mass regardless of a dwarf's baryonic luminosity is universal (e.g., Adén et al. 2009).

All of dEs and dSphs studied in detail so far reveal varying fractions of old populations (Grebel \& Gallagher 2004; Da Costa et al. 2010) that become dominant at low galactic 
masses, while intermediate-age populations ( $>1$ Gyr) are prominent at higher galactic masses. Still, no two dwarfs share the same evolutionary history or detailed abundance properties (Grebel 1997). Population gradients are found in many early-type dwarfs. Where present, younger and/or more metal-rich populations tend to be more centrally concentrated (e.g., Harbeck et al. 2001; Lisker et al. 2006; Crnojević et al. 2010).

The Galactic dSphs reach solar $[\alpha / \mathrm{Fe}]$ ratios at much lower $[\mathrm{Fe} / \mathrm{H}]$ than typical Galactic halo stars, which suggests low star formation rates, the loss of metals and supernova ejecta, and/or a larger contribution from SNe Ia (e.g., Shetrone et al. 2001). Abundance spreads of 1 dex in $[\mathrm{Fe} / \mathrm{H}]$ and more are common. The scatter in $\alpha$ element abundance ratios at a given metallicity underlines the inhomogeneous, localized enrichment in the early-type dwarfs, another characteristic expected of slow, stochastic star formation and low star formation efficiencies (Koch et al. 2008a, 2008b; Marcolini et al. 2008).

\section{References}

Adén, D., et al. 2009, ApJ, 706, 150

Balogh, M. L., et al. 2004, ApJ, 615, L101

Barnes, J. E. 2002, MNRAS, 333, 481

Barth, A. J., Martini, P., Nelson, C. H., \& Ho, L. C. 2003, ApJ, 594, L95

Bell, E. F., et al. 2008, ApJ, 680, 295

Bensby, T., Feltzing, S., Lundström, I., \& Ilyin, I. 2005, A\&A A, 433, 185

Bothwell, M. S., Kennicutt, R. C., \& Lee, J. C. 2009, MNRAS, 400, 154

Bournaud, F., Elmegreen, B. G., \& Elmegreen, D. M. 2007, ApJ, 670, 237

Bournaud, F., Elmegreen, B. G., \& Martig, M. 2009, ApJ, 707, L1

Brook, C. B., Kawata, D., Gibson, B. K., \& Freeman, K. C. 2004, ApJ, 612, 894

Bush, S. J., et al. 2010, ApJ, 713, 780

Carollo, D., et al. 2007, Nature, 450, 1020

Cignoni, M. \& Tosi, M. 2010, Adv. Ast., Vol. 2010, 1

Clemens, M. S., Bressan, A., Nikolic, B., \& Rampazzo, R. 2009a, MNRAS, 392, L35

Clemens, M. S., et al. 2009b, MNRAS, 392, 982

Cooper, M. C., et al. 2008, MNRAS, 383, 1058

Cowie, L. L., Songaila, A., Hu, E. M., \& Cohen, J. G. 1996, AJ, 112, 839

Crnojević, D., Grebel, E. K., \& Koch, A. 2010, A\&\&A, 516, A85

Da Costa, G. S., Rejkuba, M., Jerjen, H., \& Grebel, E. K. 2010, ApJ, 708, L121

Dalcanton, J. J. \& Bernstein, R. A. 2002, AJ, 124, 1328

Dekel, A. \& Woo, J. 2003, MNRAS, 344, 1131

Dressler, A. 1980, ApJ, 236, 351

Dressler, A. \& Gunn, J. E. 1983, ApJ, 270, 7

Donato, F., et al. 2009, MNRAS, 397, 1169

Dong, S., Lin, D. N. C., \& Murray, S. D. 2003, ApJ, 596, 930

D’Onghia, E., Besla, G., Cox, T. J., \& Hernquist, L. 2009, Nature, 460, 605

Elvis, M., Marengo, M., \& Karovska, M. 2002, ApJ, 567, L107

Elmegreen, B. G. \& Elmegreen, D. M. 1986, ApJ, 311, 554

Elmegreen, D. M., Elmegreen, B.G., Ravindranath, S., \& Coe, D. A. 2007, ApJ, 658, 763

Elmegreen, B. G., Bournaud, F., \& Elmegreen, D.M. 2008, ApJ, 688, 67

Elmegreen, B. G., Elmegreen, D. M., Fernandez, M. X., \& Lemonias, J. J. 2009, ApJ, 692, 12

Fan, X., et al. 2001, AJ, 122, 2833

Fan, X. 2006, New Astron. Revs, 50, 665

Finkelstein, S. L., et al. 2010, ApJ, 719, 1250

Fisher, D. B., Drory, N., \& Fabricius, M. H. 2009, ApJ, 697, 630

Freeman, K. \& Bland-Hawthorn, J. 2002, Ann. Rev. Astron. Astroph., 40, 487

Freudling, W., Corbin, M. R., \& Korista, K. T. 2003, ApJ, 587, L67

Gallazzi, A., Brinchmann, J., Charlot, S., \& White, S. D. M. 2008, MNRAS, 383, 1439 
Geisler, D., Wallerstein, G., Smith, V. V., \& Casetti-Dinescu, D. I. 2007, PASP, 119, 939

Gilmore, G. \& Reid, N. 1983, MNRAS, 202, 1025

Gilmore, G., et al. 2007, ApJ, 663, 948

Glatt, K., et al. 2008, AJ, 136, 1703

Governato, F., et al. 2009, MNRAS, 398, 312

Goto, T., et al. 2003, MNRAS, 346, 601

Goto, T. 2005, MNRAS, 357, 937

Grebel, E. K. 1997, Rev. Mod. Astron., 10, 29

Grebel, E. K. 2000, in Star formation from the small to the large scale, ESLAB Symposium 33, ESA SP 445, eds. F. Favata, A. Kaas, \& A. Wilson (ESA: Noordwijk), 87

Grebel, E. K. 2003, ApESSSS, 284, 947

Grebel, E. K. 2004, in Origin and Evolution of the Elements, Carnegie Observatories Astrophysics Series, Vol. 4, eds. A. McWilliam \& M. Rauch (Cambridge: CUP), 237

Grebel, E. K., Gallagher, J. S., III, \& Harbeck, D. 2003, AJ, 125, 1926

Grebel, E. K. \& Gallagher, J. S., III 2004, ApJ, 610, L89

Harbeck, D., et al. 2001, AJ, 122, 3092

Hartley, W. G., et al. 2010, MNRAS, 1089

Helmi, A. 2008, A\&ARv, 15, 145

Hensler, G., Theis, C., Gallagher, J. S., III 2004, A\&A, 426, 25

Hunter, D. A. 1997, PASP, 109, 937

Hunter, D. A. \& Elmegreen, B. G. 2004, $A J, 128,2170$

Ibata, R. A., Gilmore, G., \& Irwin, M. J. 1994, Nature, 370, 194

Ibata, R., Irwin, M., Lewis, G., Ferguson, A. M. N., \& Tanvir, N. 2001, Nature, 412, 49

Iwamuro, F., et al. 2004, ApJ, 614, 69

Jiang, L., et al. 2010, Nature, 464, 380

Juneau, S., et al. 2005, ApJ, 619, L135

Johnston, K. V., et al. 2008, ApJ, 689, 936

Karachentsev, I. D., et al. 2002a, $A \& A$, 385, 21

Karachentsev, I. D., et al. 2002b, $A \mathscr{\xi} A, 383,125$

Karachentsev, I. D., et al. 2003a, A\&A, 398, 467

Karachentsev, I. D., et al. 2003b, A\&AA, 404, 93

Kautsch, S. J., Grebel, E. K., Barazza, F. D., \& Gallagher, J. S., III 2006, A\& $A$, 445, 765

Kennicutt, R. C., Jr. 1998, Ann. Rev. Astron. Astroph., 36, 189

Klessen, R. S., Grebel, E. K., \& Harbeck, D. 2003, ApJ, 589, 798

Kniazev, A. Y., et al. 2003, ApJ, 593, L73

Kniazev, A. Y., et al. 2005, AJ, 130, 1558

Kniazev, A. Y., et al. 2009, MNRAS, 400, 2054

Koch, A., et al. 2007a, AJ, 134, 566

Koch, A., et al. 2007b, ApJ, 657, 241

Koch, A., et al. 2008a, AJ, 135, 1580

Koch, A., McWilliam, A., Grebel, E. K., Zucker, D. B., \& Belokurov, V. 2008b, ApJ, 688, L13

Kravtsov, A. V., Gnedin, O. Y., \& Klypin, A. A. 2004, ApJ, 609, 482

Kormendy, J. \& Kennicutt, R. C., Jr. 2004, Ann. Rev. Astron. Astroph., 42, 603

Kroupa, P. 2002, MNRAS, 330, 707

Kunth, D. \& Östlin, G. 2000, $A \& A R v, 10,1$

Kurk, J. D., et al. 2007, ApJ, 669, 32

Lee, J. C., et al. 2007, ApJ, 671, L113

Lisker, T., Grebel, E. K., \& Binggeli, B. 2006a, AJ, 132, 497

Lisker, T., Glatt, K., Westera, P., \& Grebel, E. K. 2006b, AJ, 132, 2432

Lisker, T., Grebel, E. K., Binggeli, B., \& Glatt, K. 2007, ApJ, 660, 1186

Madau, P., et al. 1996, MNRAS, 283, 1388

Maiolino, R., et al. 2004, Nature, 431, 533

Marchesini, D., et al. 2009, ApJ, 701, 1765

Marcolini, A., D'Ercole, A., Battaglia, G., \& Gibson, B.K. 2008, MNRAS, 386, 2173 
Martell, S. L. \& Grebel, E. K. 2010, A\&A, in press (arXiv:1005.4070)

Martínez-Delgado, D., et al. 2008, ApJ, 689, 184

Martínez-García, E. E., González-Lópezlira, R. A., \& Bruzual, G. 2009, ApJ, 694, 512

Matteucci, F., \& Recchi, S. 2001, ApJ, 558, 351

Mayer, L., Mastropietro, C., Wadsley, J., Stadel, J., \& Moore, B. 2006, MNRAS, 369, 1021

Meléndez, J., et al. 2008, A\&GA, 484, L21

Mieske, S., Hilker, M., \& Infante, L. 2004, A\& A, 418, 445

Minniti, D. \& Zijlstra, A. A. 1996, ApJ, 467, L13

Newberg, H. J., et al. 2002, ApJ, 569, 245

Noguchi, M. 1999, ApJ, 514, 77

Nordström, B., et al. 2004, A\& $A$ A, 418, 989

Odenkirchen, M., et al. 2001a, ApJ, 548, L165

Odenkirchen, M., et al. 2001, AJ, 122, 2538

Oemler, A., Jr. 1974, ApJ, 194, 1

Panter, B., Jimenez, R., Heavens, A. F., \& Charlot, S. 2008, MNRAS, 391, 1117

Parodi, B. R. \& Binggeli, B. 2003, A\& A, 398, 501

Poggianti, B. M., et al. 2008, ApJ, 684, 888

Poggianti, B. M., et al. 2009, ApJ, 697, L137

Poggianti, B. M., et al. 2010, MNRAS, 405, 995

Postman, M. \& Geller, M. J. 1984, ApJ, 281, 95

Richer, M., McCall, M. L., \& Stasinska, G. 1998, A\& $A$, 340, 67

Rocha-Pinto, H. J., Scalo, J., Maciel, W. J., \& Flynn, C. 2000, A\&A, 358, 869

Roškar, R., Debattista, V. P., Quinn, T. R., Stinson, G. S., \& Wadsley, J. 2008, ApJ, 684, L79

Roye, E. W. \& Hunter, D. A. 2000, AJ, 119, 1145

Sales, L. V., et al. 2009, MNRAS, 400, L61

Samland, M. \& Gerhard, O. E. 2003, A\&A, 399, 961

Schawinski, K., et al. 2007, ApJS, 173, 512

Serjeant, S., et al. 2010, A\&A, 518, L7

Seymour, N., et al. 2008, MNRAS, 386, 1695

Sheth, R. K., Jimenez, R., Panter, B., \& Heavens, A. F. 2006, ApJ, 650, L25

Shetrone, M. D., Côté, P., \& Sargent, W. L. W. 2001, ApJ, 548, 592

Stanimirovic, S., et al. 1999, MNRAS, 302, 417

Thomas, D. \& Davies, R. L. 2006, MNRAS, 366, 510

Thomas, D., Maraston, C., Schawinski, K., Sarzi, M., \& Silk, J. 2010, MNRAS, 404, 1775

Todini, P. \& Ferrara, A. 2001, MNRAS, 325, 726

Tosi, M., Greggio, L., Marconi, G., \& Focardi, P. 1991, AJ, 102, 951

Yamauchi, C., Yagi, M., \& Goto, T. 2008, MNRAS, 390, 383

Yanny, B., et al. 2003, ApJ, 588, 824

Young, L. M. \& Lo, K. Y., 1997, ApJ, 490, 710

van den Bergh, S. 1994, ApJ, 428, 617

van den Bergh, S. 2002, PASP, 114, 797

van der Marel, R. P. 2001, AJ, 122, 1827

van Zee, L., Haynes, M. P., Salzer, J. J., \& Broeils, A. H. 1997, AJ, 113, 1618

Vollmer, B., Cayatte, V., Balkowski, C., \& Duschl, W. J. 2001, ApJ, 561, 708

Walker, M. G., et al. 2007, ApJ, 667, L53

Walter, F., et al. 2004, ApJ, 615, L17

Whitmore, B. C., Gilmore, D. M., \& Jones, C. 1993, ApJ, 407, 489

Wilkinson, M. I., et al. 2004, ApJ, 611, L21

Wolf, J., et al. 2010, MNRAS, 406, 1220

Wyse, R. F. G., et al. 2006, ApJ, 639, L13

Zaritsky, D., Harris, J., Grebel, E. K., \& Thompson, I. B. 2000, ApJ, 534, L53

Zibetti, S., White, S. D. M., Brinkmann, J. 2004, MNRAS, 347, 556

Zolotov, A., et al. 2009, ApJ, 702, 1058

Zucker, D. B., et al. 2004, ApJ, 612, L117 